\title{
Design of A Continuous and Automatic Controlled Congestion Irrigator Based On Color Sensor
}

\author{
Siyuan Chen \\ School of North China Electric Power University, Baoding 071000, China;
}

18179426506@163.com

Keywords: TCS230 Color Sensor, Congestion irrigator, Micro pressure sensor, T300-S11 Longer peristaltic pump, Automatic control

\begin{abstract}
This article mainly introduces the design of the continuous and automatic controlled congestion irrigator based on STC230 color sensor, which contains hardware design and software design. There are three aspects being taken account of in hardware design. They are the power supply, the whole single processing and the module of voltage-to-current converter. Especially the mothed to convert the voltage signal ranging from $0 \mathrm{~V}$ to $3.3 \mathrm{~V}$ into current signal ranging from $4 \mathrm{~mA}$ to $20 \mathrm{~mA}$, is of significance to present industry. As for software design, on the one hand, it is essential to gain the frequency signal and the voltage signal by programming the microcontroller-K60. On the other hand, the control algorithm is also of the essence. PID control with dead zone is utilized to control the T300-S11 longer peristaltic pump to work steadily and intelligently.
\end{abstract}

\section{Introduction}

As our social ages, the incidence of prostate and bladder disease is increasing every year, continuous bladder irrigation is an important treatment at the pre-operation and postoperation for patients with prostatic hyperplasia, bladder cancer, urinary tract stones and other diseases, and its main roles include: prevention and treatment of infections, bleeding, catheter blockage, urinary retention and adjuvant treatment of bladder tumors. ${ }^{[1]}$ The traditional bladder irrigation is manually adjusted the speed of flashing based on the color of the drainage fluid, in other words, it should accelerate processing speed while the color is getting darker and slow down the processing speed if it is light in color. By this way, it needs the patient's family or caregiver to observe them all the time, which is heavy workload. Furthermore, it is controlled by human's experience, which is imprecise and inconvenient.

In allusion to traditional bladder irrigation, we come up with a continuous and automatic controlled congestion irrigation by using the TCS230 color sensor, a microcontroller, T300-S11 Longer peristaltic pump and a pressure sensor. The color sensor detects the change of fluid color, then the microcontroller gives an accurate value to control the pump timely via the output of the color sensor, at the same time, the pressure sensor inspects the pressure in patient's bladder all the while to guarantee patient's safety. The controller utilize the color sensor and a peristaltic pump, which can effectively reduce costs, improve the degree of control accuracy and the level of patient care.

\section{Hardware Design}

\section{Part 1: Power Supply}

There are many power supplies that we need to consider. First of all, the peristaltic pump needs DC $24 \mathrm{~V}$ to work, so we find a power converter which can transform AC $220 \mathrm{~V}$ into DC $24 \mathrm{~V}$. Besides, the DC $24 \mathrm{~V}$ can satisfy different power requirements for other electronic modules by different regulator chips. The framework of specific power supply shows in figure 1. 


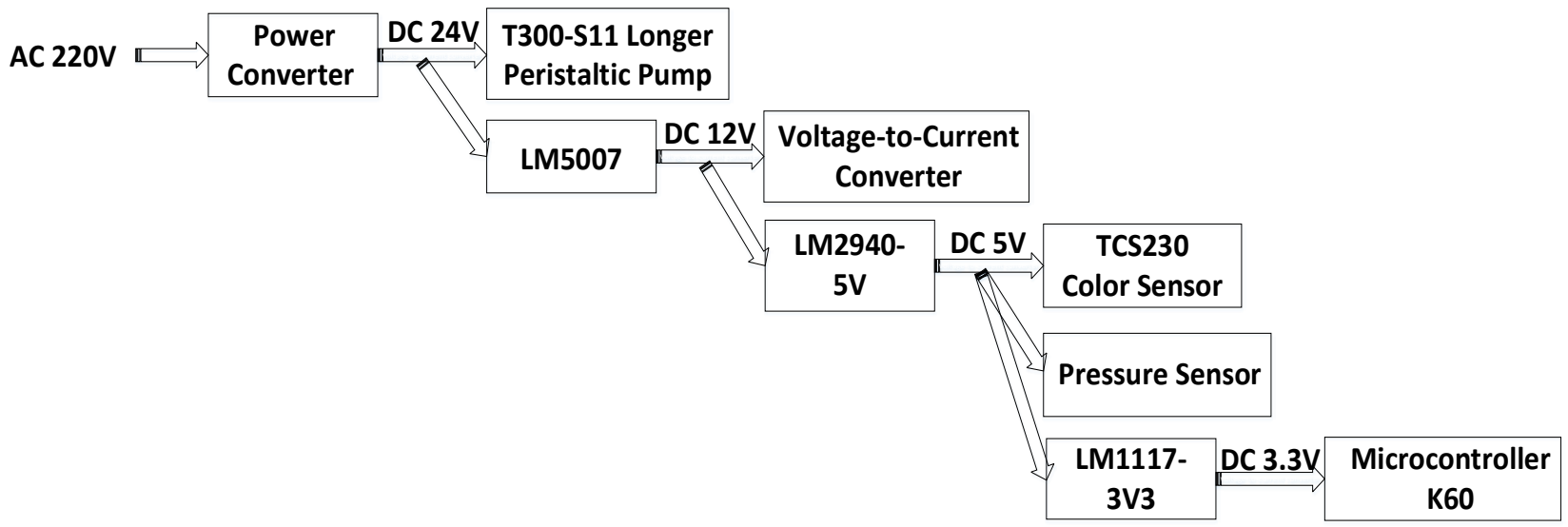

Figure 1. The Framework of Specific Power Supply

In Figure 1, LM5007 ${ }^{[2]}$ is a high voltage step down switching regulator, LM2940-5V is a chip that can step down the voltage in $5 \mathrm{~V}$, analogously, LM1117-3V3 is a regulator chip to supply the microcontroller-K60.

\section{Part 2: Single Processing}

In order to control the syringe speed of the peristaltic pump, there is a current single ranging from $4 \mathrm{~mA}$ to $20 \mathrm{~mA}$ that is required to be input. However, it is scarcely possible to be accomplished by microcontroller alone. Under this circumstance, we make use of the D/A function of K60 to get an analog voltage as its output, then convert it into current single to the pump by means of a voltage-to-current converter. The schematic diagram of the whole control system is shown in Figure 2.

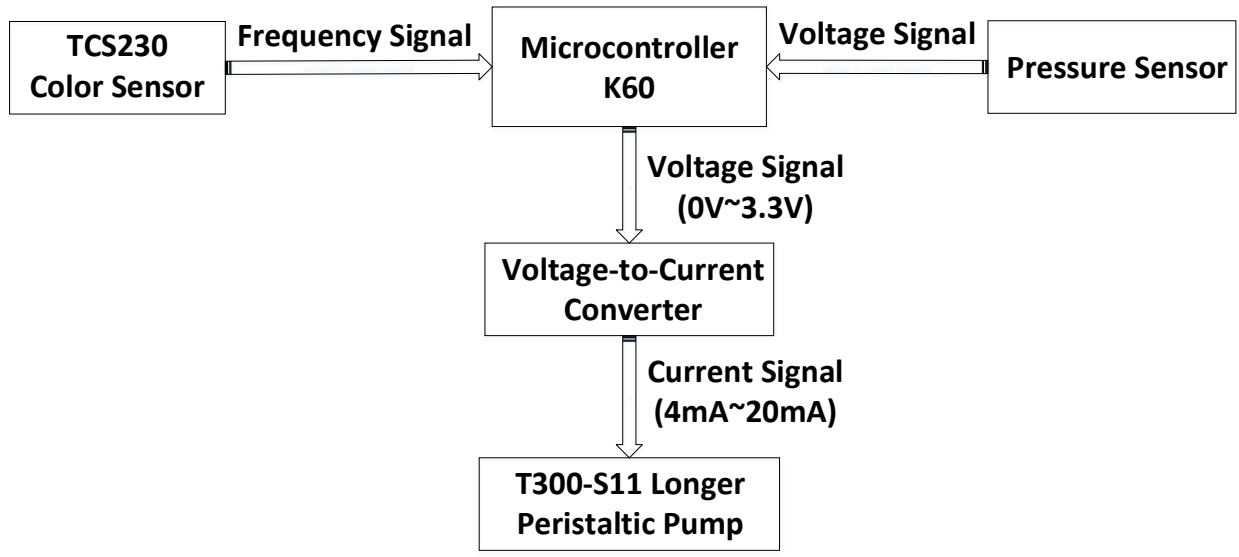

Figure. 2 The Schematic Diagram of The Whole Control System

As shown in the above figure, it is clearly to see that K60 gets the frequency signal to judge the depth of fluid color from the color sensor, then output an optimum voltage signal to control the syringe speed of the peristaltic pump. Meanwhile, pressure sensor is checking patients' bladder pressure all the time, which plays a significant role to protect patients' safety.

\section{Part 3: Voltage-to-current Converter Module}

As everyone knows, the most widely used in industry to transmit signals is the analog current signal with the scope of $4 \mathrm{~mA}$ to $20 \mathrm{~mA}$, it is because the increase of transmission distance can degrade the voltage signal while current signal is stable, people convert it to current signal for avoiding loss of signal.

\section{Software Design}

Every microcontroller has the similar processing function, K60 runs the program faster. In the aspect of software programming, as a crucial link of all the module, K60 has an obligation to consider 
all the signal processing. What's more, we need to code the appropriate control algorithm, that can make K60 computes accommodative control value to achieve a continuous and automatic control.

\section{Part 1: TCS230 Color Sensor}

TCS230 color sensor is a new sensor that the TAOS (Texas Advanced Optoelectronic Solutions) company put forward recently to detect color, it can not only realize the recognition and detection of primary colors (RGB), but also output frequency signal which is easy to deal with. Compared with the traditional color sensor, STC230 color sensor has many good qualities. For instance, its reaction speed is fast, we can choose and set the specific color channel, in addition, its output is a digital frequency signal, and it has the strong anti-interference ability. ${ }^{[3]}$

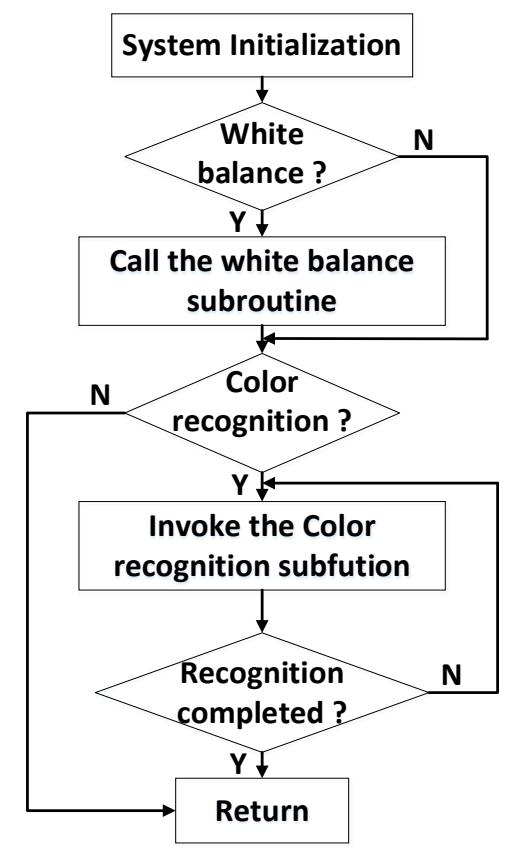

Figure 3. The software flow pattern

See from Fig. 3 above, we can analyse that, in the program flow ${ }^{[4]}$, system initialization is responsible for setting the microcontroller's timer/counter function, choosing output scaling factor of TCS230, setting the enable output and communication parameters. After that, check whether it's necessary to adjust the white balance. If so, call the white balance subroutine for adjustment. Otherwise, you can go to the next step, judge the necessity of color recognition. If you don't have to take the color identification, return; if not, invoke the subfunction that is for color recognition until it is completed.

\section{Part 2: Pressure Sensor}

There are already plenty of research on bladder pressure measurement, we can find the normal range of bladder pressure through some detail measuring points. Such as bladder leak point pressure ${ }^{[5]}$, generally, it is normal if the pressure value is less than $40 \mathrm{~cm} \mathrm{H}_{2} \mathrm{O}$, and the normal range of detrusor pressure ${ }^{[6]}$ is from $41.0 \mathrm{~cm} \mathrm{H}_{2} \mathrm{O}$ to $61.1 \mathrm{~cm} \mathrm{H}_{2} \mathrm{O}$.

According to the unit conversion formula:

$$
1 \mathrm{~cm} \mathrm{H}_{2} \mathrm{O}=0.098 \mathrm{kPa}
$$

Then we can obtain the normal bladder leak point pressure is $3.92 \mathrm{kPa}$, and the normal range of detrusor pressure is from $4.018 \mathrm{kPa}$ to $5.9878 \mathrm{kPa}$.

Pressure sensors can convert a variety of pressure signals into corresponding voltage signals, and then these signals are amplified to be an input to the microcontroller. The maximum measurable voltage value is $40 \mathrm{kPa}$, then we can derive the normal allowed voltage value. The relationship between voltage and pressure is shown below. 


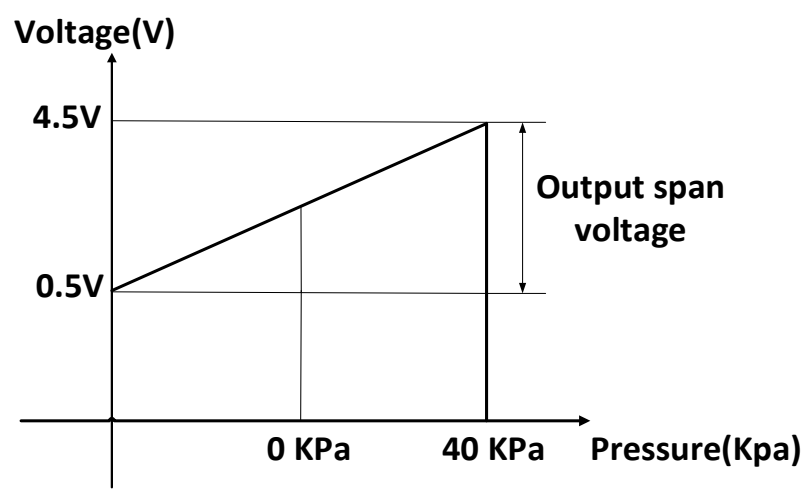

Figure 4. the Relationship between Voltage and Pressure

\section{Part 3: Control System}

As figure 5 shows, $\mathrm{r}$ represents the fluid color setpoint, color sensor and pressure sensor gives a measured value respectively as feedback signals to this system. We apply PID control with deadzone to this system, which can make sure the right control signal. Finally we can tune the applicable PID parameters through constant debugging.

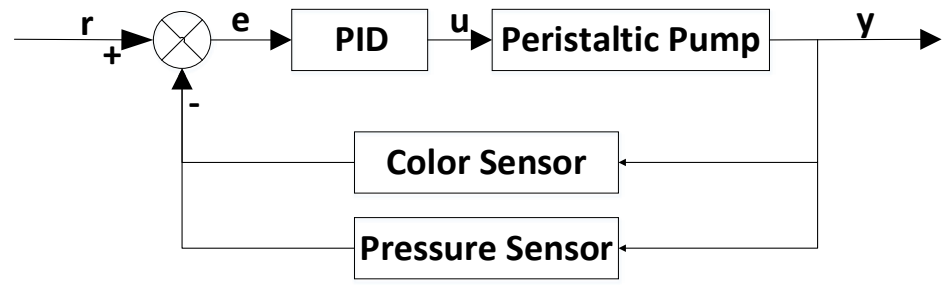

Figure 5. The Control System

\section{Conclusion}

Compared with the traditional bladder irrigation, the continuous and automatic controlled congestion irrigator based on color sensor we design has some prominent innovative characteristics. First of all, the color sensor substitutes human eyes, it quantifies the color depth of the drainage fluid as a frequency signal, which is accurate and jarless. Secondly, the microcontroller controls revolving speed of peristaltic pump via ordinary but practical control algorithm. What's more, in order to improve the safety of using device, pressure sensor is monitoring patients' bladder pressure all the time. In conclusion, the continuous and automatic controlled congestion irrigator based on color sensor is more precise, more stable and costs less, which can be helpful for our modern medical career.

\section{References}

[1] ZHANG, Aili, HONG, Shentao, et, al. A clinical evaluation of gastrocystoplasty[J]. Chinese Journal of Urology, 1999, 20(8): 491-493

[2] http://pdf1.alldatasheet.com/datasheet-pdf/view/84422/NSC/LM5007.html

[3] Yang Xianxian, Research of color detection based on TCS230[D]. Master, 2011

[4] http://www.symcukf.com/

[5] Su, ping. Significance of urinary bladder leak point pressure in patients with neurogenic bladder and upper urinary tract damage[J]. Chinese Journal of Clinical Medicine, 2010, 38(7): 29-30

[6] ZHANG, Aili, HONG, Shentao, et, al. A clinical evaluation of gastrocystoplasty[J]. Chinese Journal of Urology, 1999, 20(8): 491-493

[7] Feasibility study on artificial anus plugging device combined with pressure sensor 